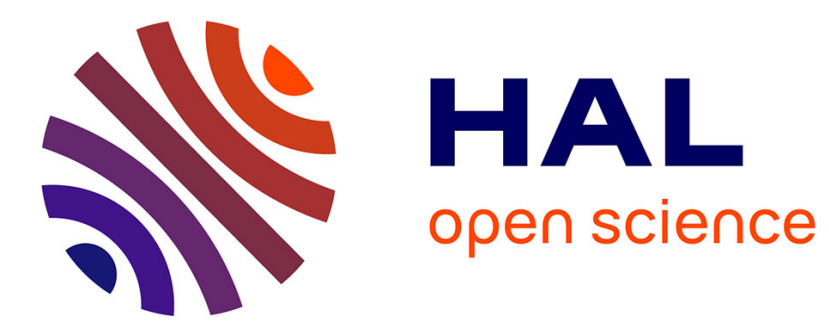

\title{
Multi-Hop Asynchronous Cooperative Diversity: Performance analysis
}

\author{
Kamel Tourki, Luc Deneire
}

\section{To cite this version:}

Kamel Tourki, Luc Deneire. Multi-Hop Asynchronous Cooperative Diversity: Performance analysis. IEEE - The 3rd International Symposium on Communications, Control and Signal Processing, Mar 2008, St. Julians, Malta. pp.1. hal-00224279

\section{HAL Id: hal-00224279 \\ https://hal.science/hal-00224279}

Submitted on 30 Jan 2008

HAL is a multi-disciplinary open access archive for the deposit and dissemination of scientific research documents, whether they are published or not. The documents may come from teaching and research institutions in France or abroad, or from public or private research centers.
L'archive ouverte pluridisciplinaire HAL, est destinée au dépôt et à la diffusion de documents scientifiques de niveau recherche, publiés ou non, émanant des établissements d'enseignement et de recherche français ou étrangers, des laboratoires publics ou privés. 


\section{Multi-Hop Asynchronous Cooperative Diversity: Performance analysis}

\author{
Kamel Tourki \\ Laboratoire I3S CNRS UNSA \\ 2000, rte des Lucioles BP 121 \\ 06903 Sophia Antipolis Cdex \\ Email: tourki@i3s.unice.fr
}

\author{
Luc Deneire \\ Laboratoire I3S CNRS UNSA \\ 2000, rte des Lucioles BP 121 \\ 06903 Sophia Antipolis Cdex \\ Email: deneire@i3s.unice.fr
}

\begin{abstract}
Mobile users with single antennas can use spatial transmission diversity through cooperative space-time encoded transmission. In this paper, we present an end-to-end performance analysis of two-hop asynchronous cooperative diversity with regenerative relays over Rayleigh block-flat-fading channels, in which a precoding frame-based scheme with packet-wise encoding is used. This precoding is based on the addition of a cyclic prefix which is implemented as a training sequence. We derive, for equal and unequal sub-channel gains, the bit-error rate and the end-to-end bit-error rate expressions for binary phase-shift keying. We also present the performance of the frame-error rate and the end-to-end frame-error rate. Finally, comparisons between three system configurations, differing by the amount of cooperation, are presented. Furthermore, simulations show that the analytical results are correct at all SNRs.
\end{abstract}

\section{INTRODUCTION}

Multiple antennas at the receiver and the transmitter are often used to combat the effects of fading in wireless communication system. However, implementing multiple antennas at the mobile stations is impractical for most wireless applications due to the limited size of the mobile unit. So, active users pool their resources to form a virtual antenna array (VAA) that realizes spatial diversity gain in a distributed fashion [1]. It is the cooperative diversity (CD) system. Cooperative transmission (without Space-Time Bloc Codes (STBC)) has been proposed in cellular networks for cooperative diversity [2], and in sensor networks for energy efficiency and fault tolerance [3]. STBC has been naturally employed for improved bandwidth efficiency besides the targeted diversity benefits [4], [5]. Unfortunately, it is difficult, and in most cases impossible, to achieve perfect synchronization among distributed transmitters. Therefore a challenge is the lack of perfect synchronization on delay and mobility of distributed transmitters. This paper focuses on performance analysis of Two-Hop asynchronous cooperative diversity system using a specific precoding, a training sequence implemented as cyclic prefix [6], [7]. This scheme offers us additional known symbols that enable best synchronization and channel estimation which reaches the Cramer-Rao Bound. We study three system configurations, differing by the amount of cooperation, using distributed STBC. In [8] we studied the performance analysis for equal sub-channel gains. This work is extended to performance analysis for unequal sub-channel gains.
In section II the system model is discussed for the three configurations. In section III, the channel and delay estimation algorithm as well as the detection scheme are discussed. The section IV shows the simulation results, and we conclude by section V.

All boldface letters indicate vectors (lower case) or matrices (upper case). The $\operatorname{tr}(\mathbf{A})$ is the trace of matrix $\mathbf{A},()^{*},()^{H}$ and ( $)^{\sharp}$ are the conjugate, the hermitian and the pseudo-inverse operators respectively. $\mathrm{E}[]$ is the Expectation operator, $\mathbf{I}_{N}$ is the identity matrix and $\mathbf{I}_{N}(L)$ is a matrix contains the $L$ latest rows on $I_{N}$. $\mathbf{O}_{N}$ is an $N \times N$ matrix with all elements equal to 0 , and we use $\mathbf{o}_{N}$ to denote an $N \times 1$ vector with all elements equal to 0 . $\mathbf{a}(n)$ is the $n^{\text {th }}$ block symbols, and $\mathbf{a}(n, k)$ is the $k^{t h}$ element of $\mathbf{a}(n)$. The Complex Gaussian distribution with mean $\mu$ and covariance matrix $\mathbf{C}$ is denoted by $\mathcal{C N}(\mu, \mathbf{C}) . \widetilde{\mathbf{d}}_{R i}(n)$ and $\widetilde{\mathbf{d}}_{R i}(n+1)$ are the decoded data by the relay terminal $R i$. $\widetilde{\mathbf{d}}(n)$ and $\widetilde{\mathbf{d}}(n+1)$ are the cooperative decoded data.

\section{SySTEM MODELS}

The general system model obeys to the multistage communication system described as follows. The mobile station $M 1$ communicating with a target $D$ via a given number of relays $M 2, R 1$ and $R 2$. Spatially adjacent mobile stations are grouped into VAAs, $M 1$ and $M 2$ form the source VAA (SVAA), $R 1$ and $R 2$ form the relay VAA (r-VAA). This system is referred to as a VAA multi-stage communication system. The symbols are replicated in space and time in a specific manner that enables the destination node to combine the received symbols in a simple manner (linear) to reap the benefits of diversity. The principle underlying this block transmission system, presented in [6], is that the block of symbols to be transmitted, instead of sending it directly, is parsed into two sub-blocks of $N$ symbols, $\mathbf{d}(n)$ and $\mathbf{d}(n+1)$. We insert $\mathrm{L}$ symbols training sequences $\mathbf{d}_{1}$ and $\mathbf{d}_{2}$ in each trail of the sub-blocks respectively, as depicted in Fig. 1. We obtain two $(N+L) \times 1$ vectors $\mathbf{s}(n)$ and $\mathbf{s}(n+1)$. These vectors are represented by relations (1) and (2).

$$
\mathbf{s}(n)=\left[\begin{array}{c}
\mathbf{d}(n) \\
\mathbf{d}_{1}
\end{array}\right]
$$




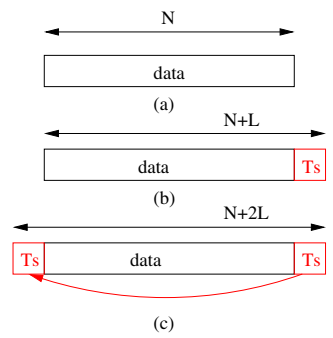

Fig. 1. A Frame description.

TABLE I

TRANSMISSION SCHEME

\begin{tabular}{|c|c|c|}
\hline & $n^{\text {th }}$ block symbols & $(n+1)^{\text {th }}$ block symbols \\
\hline ant $\mathbf{t} \mathbf{x}_{1}$ & $\mathbf{F}_{P} \times \mathbf{s}(n)$ & $-\left(\mathbf{F}_{P} \times \mathbf{s}_{v}(n+1)\right)^{*}$ \\
ant $\mathbf{t x}_{2}$ & $\mathbf{F}_{P} \times \mathbf{s}(n+1)$ & $\left(\mathbf{F}_{P} \times \mathbf{s}_{v}(n)\right)^{*}$ \\
\hline
\end{tabular}

$$
\mathbf{s}(n+1)=\left[\begin{array}{c}
\mathbf{d}(n+1) \\
\mathbf{d}_{2}
\end{array}\right]
$$

In order to facilitate the detection procedure as it will be shown in section III, we use time reversal matrices $\mathbf{T}$ and $\mathbf{T}_{s}$ as linear precoding to obtain $\mathbf{s}_{v}(n)$ and $\mathbf{s}_{v}(n+1)$

$$
\begin{aligned}
\mathbf{s}_{v}(n) & =\left[\begin{array}{c}
\mathbf{T d}(n) \\
\mathbf{T}_{s} \mathbf{d}_{1}
\end{array}\right] \\
\mathbf{s}_{v}(n+1) & =\left[\begin{array}{c}
\mathbf{T d}(n+1) \\
\mathbf{T}_{s} \mathbf{d}_{2}
\end{array}\right]
\end{aligned}
$$

Our method consists in inserting a cyclic prefix between any two successive blocks which is depicted in (c) in Fig. 1. This operation is done when pre-multiplying in the left by $\mathbf{F}_{p}$, so $\mathbf{s}(n)$ and $\mathbf{s}_{v}(n)$ are extended to $N+2 L$ symbols. Therefore a distributed space time coding is applied and the transmitted frames $\mathbf{s}_{1}[n]$ and $\mathbf{s}_{2}[n]$ are formed as follows

$$
\begin{gathered}
\mathbf{s}_{1}[n]=\left[\begin{array}{c}
\mathbf{F}_{p} \mathbf{s}(n) \\
-\left(\mathbf{F}_{p} \mathbf{s}_{v}(n+1)\right)^{*}
\end{array}\right] \\
\mathbf{s}_{2}[n]=\left[\begin{array}{c}
\mathbf{F}_{p} \mathbf{s}(n+1) \\
\left(\mathbf{F}_{p} \mathbf{s}_{v}(n)\right)^{*}
\end{array}\right]
\end{gathered}
$$

where

$$
\mathbf{F}_{p}=\left[\begin{array}{c}
I_{N+L}(L) \\
I_{N+L}
\end{array}\right]
$$

$\mathbf{T}$ and $\mathbf{T}_{s}$ are represented in Fig. 2. Each link of this system is
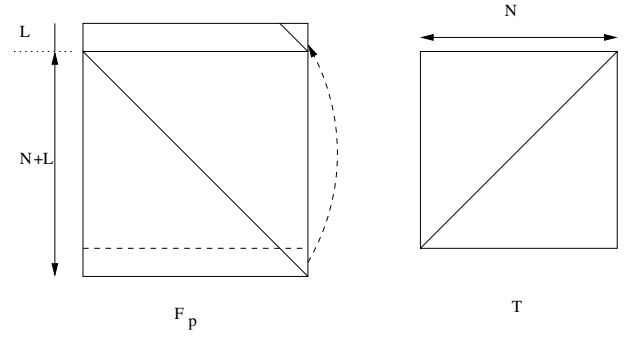

Fig. 2. The precoding matrices considered a point-to-point one way communication link, and the channel is assumed Rayleigh block-flat-fading, constant during the transmission of one frame and independent from frame to frame. We study the performance analysis of two-hop asynchronous cooperative diversity in three configurations [8] listed below. But in the first hop, there are no particularities, every relay receives a summation of the signals of the two active mobiles in the source VAA after they travel through different paths in the channel. These channel paths induce different delays, attenuations and phase shifts. Therefore, these transmission delays and channels can be estimated from training sequences. We define $\tau_{1}$ and $\tau_{2}$ respectively as the arrival time of the first and the second signals received by the mobile station $R 1$ and we define $\tau_{3}$ and $\tau_{4}$ respectively as the arrival time of the first and the second signals received by the mobile station $R 2$. We assume without loss of generality that $\tau_{1} \leq \tau_{2}$ and $\tau_{3} \leq \tau_{4}$, and the analysis is done for $R 1$. The received signal by $R 1$ is given by

$$
\mathbf{r}=\mathcal{A}(\tau) \mathbf{X h}+\mathbf{b}
$$

where the total noise vector $\mathbf{b} \sim \mathcal{C N}\left(\mathbf{0}, N_{0} \mathbf{I}_{2 N+4 L}\right)$ and

$$
\begin{gathered}
\mathcal{A}(\tau)=\left[\begin{array}{lll}
\mathbf{O}_{2 N+4 L} & \mathbf{I}_{2 N+4 L} & \Gamma
\end{array}\right] \\
\mathbf{X}=\left[\begin{array}{cc}
\mathbf{s}_{1}[n-1] & \mathbf{o}_{2 N+4 L} \\
\mathbf{s}_{1}[n] & \mathbf{o}_{2 N+4 L} \\
\mathbf{o}_{2 N+4 L} & \mathbf{s}_{2}[n-1] \\
\mathbf{o}_{2 N+4 L} & \mathbf{s}_{2}[n]
\end{array}\right] \\
\mathbf{h}=\left[\begin{array}{l}
h_{1} \\
h_{2}
\end{array}\right]
\end{gathered}
$$

In the equation above, $h_{1,2} \sim \mathcal{C N}\left(0, \gamma_{1,2}\right)$ are the complex scalar channel parameters, $\mathbf{X}$ is the matrix obtained by stacking two consecutive frames from each transmitter. $\Gamma$ and $\Psi$ with size $(2 N+4 L) \times(2 N+4 L)$ account for the asynchronism between the two signals, and are expressed respectively as

$$
\begin{gathered}
\Gamma=\left[\begin{array}{cc}
\mathbf{O}_{L_{\tau} \times\left(2 N+4 L-L_{\tau}\right)} & \mathbf{I}_{L_{\tau}} \\
\mathbf{O}_{\left(2 N+4 L-L_{\tau}\right) \times\left(2 N+4 L-L_{\tau}\right)} & \mathbf{O}_{\left(2 N+4 L-L_{\tau}\right) \times\left(L_{\tau}\right)}
\end{array}\right] \\
\Psi=\left[\begin{array}{cc}
\mathbf{O}_{L_{\tau} \times\left(2 N+4 L-L_{\tau}\right)} & \mathbf{O}_{L_{\tau}} \\
\mathbf{I}_{2 N+4 L-L_{\tau}} & \mathbf{O}_{\left(2 N+4 L-L_{\tau}\right) \times\left(L_{\tau}\right)}
\end{array}\right]
\end{gathered}
$$

where $L_{\tau}=\tau_{2}-\tau_{1}$ is the relative delay which is bounded by $L$. We can remark that in synchronous case, $\Gamma_{s y n}=\mathbf{O}_{2 N+4 L}$ and $\Psi_{\text {syn }}=\mathbf{I}_{2 N+4 L}$. We remember that the cooperative links in the source VAA and the relay VAA are assumed error-free due to the short communication distances between the mobiles of the same VAA compared to the inter-VAA distances. Any of the relays receives signals and functions as follows.

$$
\begin{aligned}
& \mathbf{r}_{1}=\mathcal{A}\left(\tau_{12}\right) \mathbf{X h}_{12}+\mathbf{b}_{1} \\
& \mathbf{r}_{2}=\mathcal{A}\left(\tau_{34}\right) \mathbf{X h}_{34}+\mathbf{b}_{2}
\end{aligned}
$$

where the total noise vectors $\mathbf{b}_{1,2} \sim \mathcal{C N}\left(\mathbf{0}, N_{0} \mathbf{I}_{2 N+4 L}\right)$ and

$$
\mathbf{h}_{12}=\left[\begin{array}{l}
h_{1} \\
h_{2}
\end{array}\right]
$$




$$
\mathbf{h}_{34}=\left[\begin{array}{l}
h_{3} \\
h_{4}
\end{array}\right]
$$

- System 1: $R 1$ and $R 2$ receive the data, estimate the channel parameters as shown in section III and decode separately the received data as shown in section III-A, before being passed onto the cooperative procedure. In order to conserve the transmitting rate, each relay cooperates by using only half of decoded frames, therefore $R 1$ uses the decoded $\widetilde{\mathbf{d}}_{R 1}(n)$ and $R 2$ uses $\widetilde{\mathbf{d}}_{R 2}(n+1)$. Therefore we re-transmit

$$
\begin{gathered}
\mathbf{s}_{1 R}[n]=\left[\begin{array}{c}
\mathbf{F}_{p} \mathbf{s}_{1}(n) \\
-\left(\mathbf{F}_{p} \mathbf{s}_{2 v}(n+1)\right)^{*}
\end{array}\right] \\
\mathbf{s}_{2 R}[n]=\left[\begin{array}{c}
\mathbf{F}_{p} \mathbf{s}_{2}(n+1) \\
\left(\mathbf{F}_{p} \mathbf{s}_{1 v}(n)\right)^{*}
\end{array}\right]
\end{gathered}
$$

where

$$
\begin{aligned}
\mathbf{s}_{1}(n) & =\left[\begin{array}{c}
\widetilde{\mathbf{d}}_{R 1}(n) \\
\mathbf{d}_{1}
\end{array}\right] \\
\mathbf{s}_{2}(n+1) & =\left[\begin{array}{c}
\widetilde{\mathbf{d}}_{R 2}(n+1) \\
\mathbf{d}_{2}
\end{array}\right] \\
\mathbf{s}_{1 v}(n) & =\left[\begin{array}{c}
\mathbf{T} \widetilde{\mathbf{d}}_{R 1}(n) \\
\mathbf{T}_{s} \mathbf{d}_{1}
\end{array}\right] \\
\mathbf{s}_{2 v}(n+1) & =\left[\begin{array}{c}
\mathbf{T} \widetilde{\mathbf{d}}_{R 2}(n+1) \\
\mathbf{T}_{s} \mathbf{d}_{2}
\end{array}\right]
\end{aligned}
$$

- System $2: R 1$ and $R 2$ receive the data, estimate the channel parameters as shown in section III and decode separately the received data as shown in section III-A. We conserve the structure of the Distributed STBC and we re-encode the decoded data without any cooperation in (r-VAA). Therefore we re-transmit

$$
\begin{gathered}
\mathbf{s}_{1 R}[n]=\left[\begin{array}{c}
\mathbf{F}_{p} \mathbf{s}_{1}(n) \\
-\left(\mathbf{F}_{p} \mathbf{s}_{v}(n+1)\right)^{*}
\end{array}\right] \\
\mathbf{s}_{2 R}[n]=\left[\begin{array}{c}
\mathbf{F}_{p} \mathbf{s}_{2}(n+1) \\
\left(\mathbf{F}_{p} \mathbf{s}_{v}(n)\right)^{*}
\end{array}\right]
\end{gathered}
$$

where

$$
\begin{aligned}
\mathbf{s}_{1}(n) & =\left[\begin{array}{c}
\widetilde{\mathbf{d}}_{R 1}(n) \\
\mathbf{d}_{1}
\end{array}\right] \\
\mathbf{s}_{2}(n+1) & =\left[\begin{array}{c}
\widetilde{\mathbf{d}}_{R 2}(n+1) \\
\mathbf{d}_{2}
\end{array}\right] \\
\mathbf{s}_{v}(n) & =\left[\begin{array}{c}
\mathbf{T} \widetilde{\mathbf{d}}_{R 2}(n) \\
\mathbf{T}_{s} \mathbf{d}_{1}
\end{array}\right] \\
\mathbf{s}_{v}(n+1) & =\left[\begin{array}{c}
\mathbf{T} \widetilde{\mathbf{d}}_{R 1}(n+1) \\
\mathbf{T}_{s} \mathbf{d}_{2}
\end{array}\right]
\end{aligned}
$$

The error performance differences between system 1 and 2 is very obvious for unequal sub-channel gains (shadowing, see Fig. 3).

- System $3: R 1$ and $R 2$ receive the data, estimate the channel parameters as shown in section III but no separately decoding is performed in (r-VAA), then an unprocessed version of the received signals are exchanged between $R 1$ and $R 2$. After cooperation, appropriate decoding is performed as shown in section III-B, the obtained information $\widetilde{\mathbf{d}}$ is then re-encoded in a distributed manner as follows, and re-transmitted to the destination

$$
\begin{gathered}
\mathbf{s}_{1}[n]=\left[\begin{array}{c}
\mathbf{F}_{p} \mathbf{s}(n) \\
-\left(\mathbf{F}_{p} \mathbf{s}_{v}(n+1)\right)^{*}
\end{array}\right] \\
\mathbf{s}_{2}[n]=\left[\begin{array}{c}
\mathbf{F}_{p} \mathbf{s}(n+1) \\
\left(\mathbf{F}_{p} \mathbf{s}_{v}(n)\right)^{*}
\end{array}\right]
\end{gathered}
$$

where

$$
\begin{aligned}
\mathbf{s}(n) & =\left[\begin{array}{c}
\widetilde{\mathbf{d}}(n) \\
\mathbf{d}_{1}
\end{array}\right] \\
\mathbf{s}(n+1) & =\left[\begin{array}{c}
\widetilde{\mathbf{d}}(n+1) \\
\mathbf{d}_{2}
\end{array}\right] \\
\mathbf{s}_{v}(n) & =\left[\begin{array}{c}
\mathbf{T} \widetilde{\mathbf{d}}(n) \\
\mathbf{T}_{s} \mathbf{d}_{1}
\end{array}\right] \\
\mathbf{s}_{v}(n+1) & =\left[\begin{array}{c}
\mathbf{T} \widetilde{\mathbf{d}}(n+1) \\
\mathbf{T}_{s} \mathbf{d}_{2}
\end{array}\right]
\end{aligned}
$$

\section{Channel and Delay Estimation Algorithm}

A maximum likelihood (ML) method for delay and channel estimation is proposed in [6], [7] and we summarize it here. We denote $\mathbf{t s}_{\mathbf{1}}$ and $\mathbf{t s}_{\mathbf{2}}$ as

$$
\begin{aligned}
& \mathbf{t s}_{\mathbf{1}}=\mathbf{T}_{s} \mathbf{d}_{1} \\
& \mathbf{t s}_{\mathbf{2}}=\mathbf{T}_{s} \mathbf{d}_{2}
\end{aligned}
$$

Therefore we define $\mathbf{S}(\tau)=\left[\mathbf{s s}_{\mathbf{1}} \mathbf{s s}_{\mathbf{2}}\right]$ where

$$
\begin{gathered}
\mathbf{s s}_{\mathbf{1}}=\left[\begin{array}{c}
\mathbf{d}_{1}(\tau+1: L) \\
-\left(\mathbf{t s}_{\mathbf{2}}\right)^{*}
\end{array}\right] \\
\mathbf{s s}_{\mathbf{2}}=\left[\begin{array}{c}
\mathbf{d}_{2} \\
\left(\mathbf{t s}_{\mathbf{1}}(1: L-\tau)\right)^{*}
\end{array}\right]
\end{gathered}
$$

For this deterministic model, we denote $\mathbf{z}(\tau)=\mathbf{r}[n, N+L+$ $\tau+1: N+3 L]$, therefore we can write

$$
\widetilde{\mathbf{h}}(\tau)=(\mathbf{S}(\tau))^{\sharp} \mathbf{z}(\tau)
$$

therefore

$$
\widehat{\tau}=\arg \min _{\tau<L}\|\mathbf{z}-\mathbf{S}(\tau) \widetilde{\mathbf{h}}(\tau)\|^{2}
$$

and

$$
\widehat{\mathbf{h}}=(\mathbf{S}(\widehat{\tau}))^{\sharp} \mathbf{z}(\widehat{\tau})
$$

The Mean Square Error (MSE) of the channel estimation is defined by

$$
M S E(h)=\mathrm{E}\left[\|\mathbf{h}-\widehat{\mathbf{h}}\|^{2}\right]
$$

We studied the Cramer-Rao Bound for this model [6] [7], we derive (see appendix VI-A)

$$
C R B=N_{0}\left[(\mathbf{S}(\tau))^{H} \mathbf{S}(\tau)\right]^{-1}
$$




\section{A. Detection scheme for one receiver}

We denote $\mathbf{r}_{a}, \mathbf{r}_{b}$ and $\mathbf{y}$ as

$$
\begin{gathered}
\mathbf{r}_{a}=\mathbf{r}[n, L+1: N+L+\tau] \\
\mathbf{r}_{b}=\mathbf{r}[n, N+3 L+1: 2 N+3 L+\tau] \\
\mathbf{y}=\left[\begin{array}{ll}
\mathbf{r}_{a}^{T} & \left.\mathbf{r}_{b}^{T}\right]
\end{array}\right.
\end{gathered}
$$

The combiner builds the following two combined signals that are sent to the maximum likelihood detector:

$$
\widetilde{\mathbf{d}}(n, k)=\widehat{\mathbf{h}}_{1}^{*}(n) \mathbf{y}(n, k)+\widehat{\mathbf{h}}_{2}(n) \mathbf{y}^{*}(n, l+1-k)
$$

$\widetilde{\mathbf{d}}(n+1, k)=\widehat{\mathbf{h}}_{2}^{*}(n) \mathbf{y}(n, \tau+k)-\widehat{\mathbf{h}}_{1}(n) \mathbf{y}^{*}(n, l-\tau+1-k)$

where $l$ is the number of bits which $\mathbf{y}$ contains.

\section{B. Detection scheme for two cooperative receivers}

We receive $\mathbf{r}_{1}$ and $\mathbf{r}_{2}$ and we denote $\mathbf{r}_{1 a}, \mathbf{r}_{1 b}$ and $\mathbf{y}_{1}$ as

$$
\begin{gathered}
\mathbf{r}_{1 a}=\mathbf{r}_{1}\left[n, L+1: N+L+\tau_{1}\right] \\
\mathbf{r}_{1 b}=\mathbf{r}_{1}\left[n, N+3 L+1: 2 N+3 L+\tau_{1}\right] \\
\mathbf{y}_{1}=\left[\begin{array}{ll}
\mathbf{r}_{1 a}^{T} & \left.\mathbf{r}_{1 b}^{T}\right]
\end{array}\right.
\end{gathered}
$$

and we denote $\mathbf{r}_{2 a}, \mathbf{r}_{2 b}$ and $\mathbf{y}_{2}$ as

$$
\begin{gathered}
\mathbf{r}_{2 a}=\mathbf{r}_{2}\left[n, L+1: N+L+\tau_{2}\right] \\
\mathbf{r}_{2 b}=\mathbf{r}_{2}\left[n, N+3 L+1: 2 N+3 L+\tau_{2}\right] \\
\mathbf{y}_{2}=\left[\begin{array}{ll}
\mathbf{r}_{2 a}^{T} & \left.\mathbf{r}_{2 b}^{T}\right]
\end{array}\right.
\end{gathered}
$$

The combiner builds the following two combined signals that are sent to the maximum likelihood detector :

$$
\begin{aligned}
\widetilde{\mathbf{d}}(n, k)= & \widehat{\mathbf{h}}_{1}^{*}(n) \mathbf{y}_{1}(n, k)+\widehat{\mathbf{h}}_{2}(n) \mathbf{y}_{1}^{*}\left(n, l_{1}+1-k\right) \\
& +\widehat{\mathbf{h}}_{3}^{*}(n) \mathbf{y}_{2}(n, k)+\widehat{\mathbf{h}}_{4}(n) \mathbf{y}_{2}^{*}\left(n, l_{2}+1-k\right)(56) \\
\widetilde{\mathbf{d}}(n+1, k)= & \widehat{\mathbf{h}}_{2}^{*}(n) \mathbf{y}_{1}\left(n, \tau_{1}+k\right)-\widehat{\mathbf{h}}_{1}(n) \mathbf{y}_{1}^{*}\left(n, \nu_{1}\right) \\
& +\widehat{\mathbf{h}}_{4}^{*}(n) \mathbf{y}_{2}\left(n, \tau_{2}+k\right)-\widehat{\mathbf{h}}_{3}(n) \mathbf{y}_{2}^{*}\left(n, \nu_{2}\right)
\end{aligned}
$$

where $l_{1}$ and $l_{2}$ are the numbers of bits which $\mathbf{y}_{1}$ and $\mathbf{y}_{2}$ contain respectively, and $\nu_{i}=l_{i}-\tau_{i}+1-k$. We remark when using time reversal matrices, the detection is facilitated and it was reduced to Alamouti scheme [9].

\section{Performance Analysis And Simulations}

For equal sub-channel gains $\gamma$, the moment generating function (MGF) of the instantaneously experienced SNR for a system with $t$ transmit antennas, $r$ receive antennas and $\lambda$ is the channel energy, can be expressed as [10]

$$
\phi_{\frac{1}{R} \frac{\lambda}{t} \frac{S}{N}}(s)=\frac{1}{\left(1-\frac{1}{R} \frac{\gamma}{t} \frac{S}{N} \times s\right)^{u}}
$$

where $R$ is the transmission rate and $u=t \times r$. The analysis in [11] allows us to express the BER for BPSK modulation in closed form as

$$
\begin{aligned}
& P s e\left(R, \gamma, \frac{S}{N}, t, r\right)=\phi_{\frac{1}{R} \frac{\lambda}{t} \frac{S}{N}}(-1)\left[\frac{1}{2 \sqrt{\pi}} \frac{\Gamma(u+1 / 2)}{\Gamma(u+1)}\right] \times \\
& { }_{2} F_{1}\left(u, 1 / 2 ; u+1 ;\left(1+\frac{1}{R} \frac{\gamma}{t} \frac{S}{N}\right)^{-1}\right)
\end{aligned}
$$

where ${ }_{2} F_{1}(a, b ; c ; x)$ is the Gauss hypergeometric function with 2 parameters of type 1 and 1 parameter of type 2 . It has been implemented using the series representations

$$
{ }_{2} F_{1}(a, b ; c ; x)=\sum_{n=0}^{\infty} \frac{(a)_{n}(b)_{n}}{(c)_{n} n !} x^{n}
$$

where

$$
(a)_{n}=\frac{\Gamma(a+n)}{\Gamma(a)}
$$

For unequal sub-channel gains, the MGF is shown to be

$$
\phi_{\frac{1}{R} \frac{\lambda}{t} \frac{S}{N}}(s)=\sum_{i=1}^{u} K_{i} \phi_{\frac{1}{R} \frac{\lambda_{i}}{t} \frac{S}{N}}(s)
$$

with constants $K_{i}$ [7] (and see appendix VI-B)

$$
K_{i}=\prod_{i^{\prime}=1, i^{\prime} \neq i}^{u} \frac{\gamma_{i}}{\gamma_{i}-\gamma_{i^{\prime}}}
$$

where $\gamma_{i}$ is the average channel gain of the $i^{t h}$ path. This allows us to derive the expression of BER in closed form where all the channel gains differ. The error rate can be expressed as

$$
P_{u c g}(u)(e)=\sum_{i=1}^{u} K_{i} P \operatorname{se}\left(R, \gamma_{i}, \frac{S}{N}, 1,1\right)
$$

For simplicity, we can note $\operatorname{Pse}\left(R, \gamma, \frac{S}{N}, t, r\right)$ and $P_{u c g}(e)$ as $P_{t, r}(e)$. Figures 3 and 4 depict the efficiency of the channel and delay estimation algorithm derived in section III.

In systems 1 and 2, the partial cooperation at the first relaying stage results in two parallel MISO channels. Assuming an error free input into the relay VAA, each of these MISO channels causes independent BERs, denoted by $P_{R 1}$ and $P_{R 2}$ respectively. If each of these MISO channels consists of channels with equal (resp unequal) average attenuations $\gamma\left(\operatorname{resp} \gamma_{i}\right)$. The second stage spans a single MISO channel with a BER $P_{D}$. To obtain the exact end-to-end BER is not trivial, as an error in the first stage may propagate to $D$; however, it may also be corrected at the next stage. Therefore, the probability that an error which occur in link $\{M 1, R 1\}$ with probability $P_{R 1}=$ 
$P_{2,1}(e)$ propagates through the O-MIMO channel spanned by $\{R 1, D\}$ and $\{R 2, D\}$ is approximated as $P_{R 1} \times \gamma /(\gamma+\gamma)$ (resp $P_{R 1} \times \gamma_{5} /\left(\gamma_{5}+\gamma_{6}\right)$ ), where the strength of the erroneous channel $\{R 1, D\}$ is normalized by the total strength of both sub-channels. To capture the probability that such an error propagates until the destination $D$, all possible paths in the network have to be found and the original probability of error weighed with the ratios between the respective path gains. The end-to-end BER can be expressed for equal sub-channel gains as

$$
P_{e 2 e}(e) \approx\left[\frac{1}{2} P_{R 1}+\frac{1}{2} P_{R 2}\right]+P_{D}
$$

where $P_{R 1}, P_{R 2}$ and $P_{D}$ are defined by (59), and for unequal sub-channel gains

$$
P_{e 2 e}(e) \approx\left[\frac{\gamma_{5}}{\gamma_{5}+\gamma_{6}} P_{R 1}+\frac{\gamma_{6}}{\gamma_{5}+\gamma_{6}} P_{R 2}\right]+P_{D}
$$

where $P_{R 1}, P_{R 2}$ and $P_{D}$ are defined by (64).

In system 3 , the full cooperation at each stage is assumed. Each of the two relaying stages experiences independent BERs. A bit from the source $M 1$ is received correctly at the target $D$ only when at all stages the bit has been transmitted correctly. The end-to-end BER can therefore be expressed as

$$
P_{e 2 e}(e)=1-\left[\left(1-P_{2,2}(e)\right)\left(1-P_{2,1}(e)\right)\right]
$$

Fig 6 depicts the end-to-end bit-error rate $P_{e 2 e}$ versus the SNR in $(\mathrm{dB})$ labeled on the system schemes exhibiting a spectral efficiency of $1 \mathrm{bit} / \mathrm{s} / \mathrm{Hz}$. Here, the solid lines represent the analytically derived $P_{e 2 e}$, whereas the markers correspond to specific points obtained by means of simulations. For all configurations, the simulations clearly corroborate the analytical results. Furthermore, the third configuration, with full cooperation at each stage, obviously enhances the end-to-end performances.

Each frame contains 288 symbols in which 224 for data. Therefore the cyclic prefix contain 16 symbols for training sequence. Figures (5) and (6) show that the difference between the system 1 and system 2 is limited in the first hop. Fig 7 depicts the end-to-end frame-error rate versus the SNR in $(\mathrm{dB})$ labeled on the system schemes exhibiting performance analysis for unequal sub-channel gains.

\section{CONClusion}

We derived the performance analysis of two-hop asynchronous cooperative diversity, where the emphasis has been on transceivers utilizing space-time block coding. The errorrates of such transceivers have been derived for three communication scenarios. As mentioned before, we have an unequal sub-channel case, and, systems 1 and 2 present a partial cooperation at the relaying stage, but system 3 is a full cooperation scenario. We remark that the differences in performances between systems 1 and 2 in the first hop are released at the destination. The performance enhancements by the full cooperation at each stage is at the expense of additional transceiver complexity to realize the cooperation; also, additional bandwidth and power are required to accomplished the relaying process.

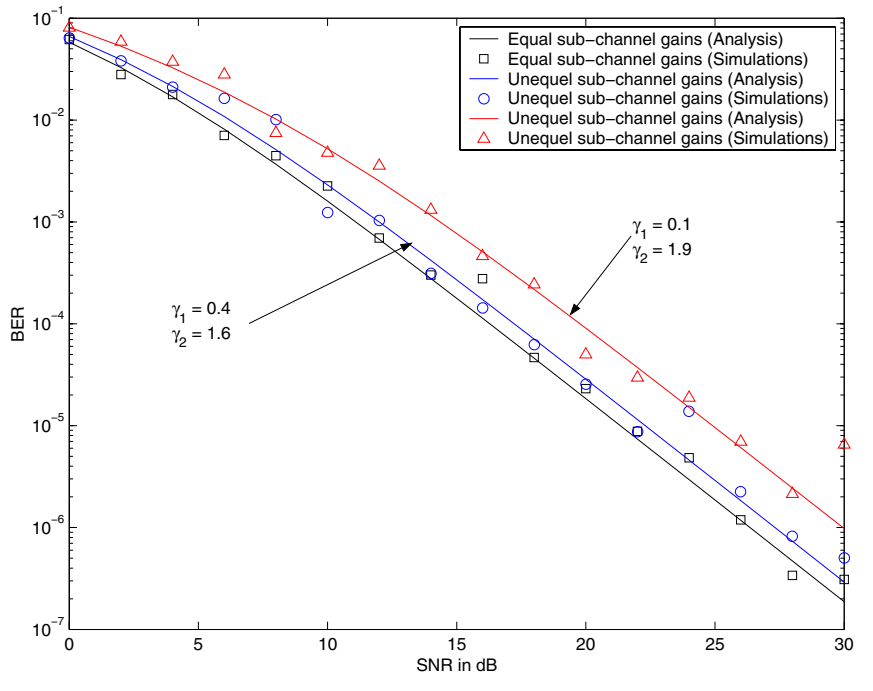

Fig. 3. BER performances of the asynchronous cooperative diversity system with channel and delay estimations for one receiver case, with equal and unequal sub-channel gains (partial cooperation).

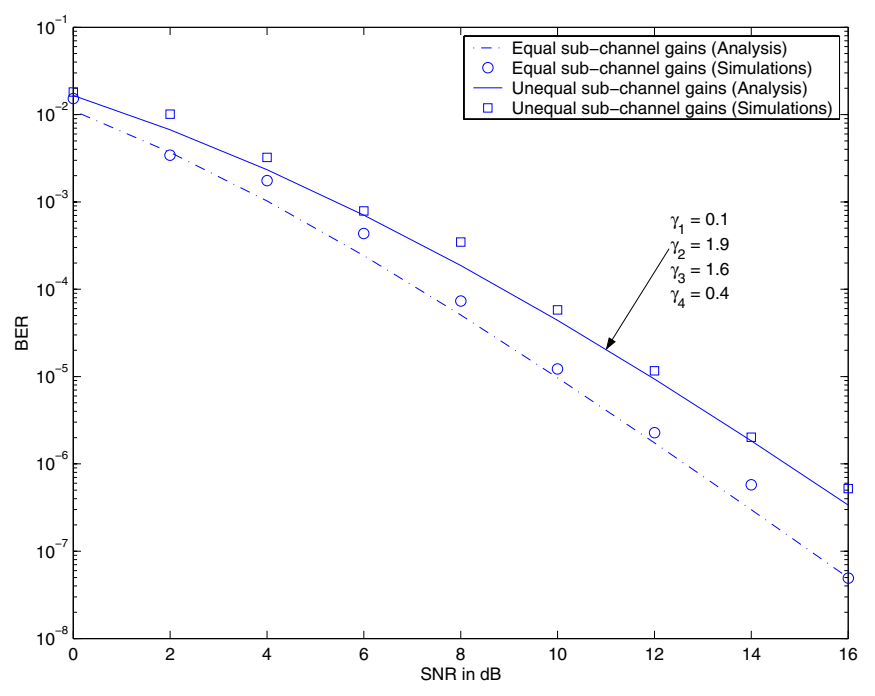

Fig. 4. BER performances of the asynchronous cooperative diversity system with channel and delay estimations for two receivers case, with equal and unequal sub-channel gains (full cooperation).

\section{APPENDIX}

\section{A. $C R B$ derivation}

The complex Fisher Information Matrix is

$$
J(h)=\frac{1}{N_{0}}\left(\frac{\partial \mathbf{r}_{s}^{H}}{\partial h^{*}}\right)\left(\frac{\partial \mathbf{r}_{s}^{H}}{\partial h^{*}}\right)^{H}
$$

Where $\mathbf{r}_{s}=\mathbf{S}(\tau) \mathbf{h}$ is the signal part of $\mathbf{r}$. Therefore, we have

$$
\begin{gathered}
\left(\frac{\partial \mathbf{r}_{s}^{H}}{\partial h^{*}}\right)=\frac{\partial \mathbf{h}^{H}(\mathbf{S}(\tau))^{H}}{\partial \mathbf{h}^{*}}=(\mathbf{S}(\tau))^{H} \\
\left(\frac{\partial \mathbf{r}_{s}^{H}}{\partial h^{*}}\right)^{H}=\mathbf{S}(\tau)
\end{gathered}
$$




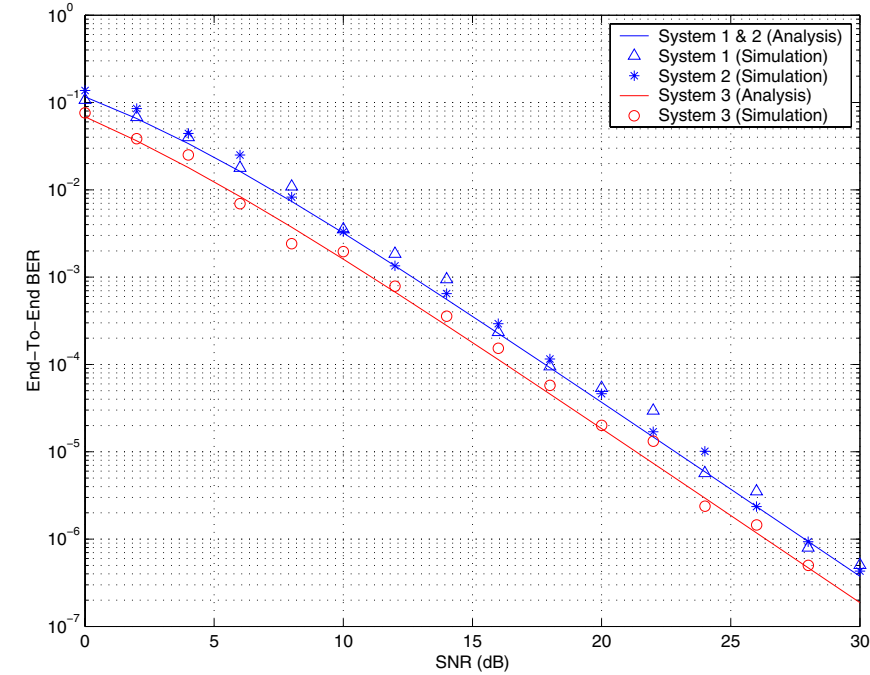

Fig. 5. Comparison between end-to-end bit-error rate of the three configurations for equal sub-channel gains.

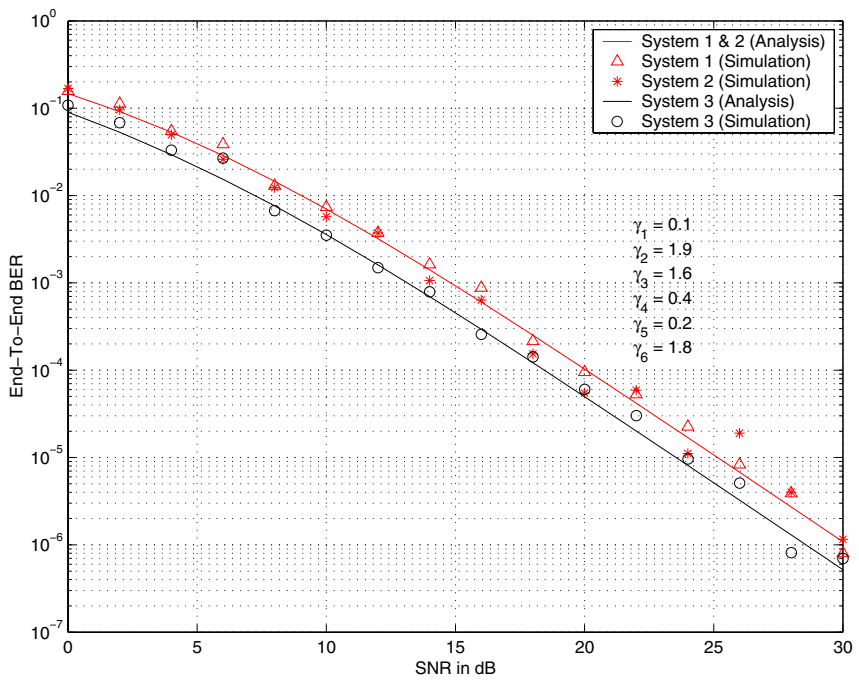

Fig. 6. Comparison between end-to-end bit-error rate of the three configurations for unequal sub-channel gains.

\section{B. Derivation of the constants $K_{i}$}

It is proven here that

$$
K_{i}=\prod_{i^{\prime}=1, i^{\prime} \neq i}^{u} \frac{\gamma_{i}}{\gamma_{i}-\gamma_{i^{\prime}}}
$$

Without loss of generality, $K_{1}$ is derived here. The fractional expansion is equated to the product expression, i.e.

$$
\sum_{i^{\prime}=1}^{u} \frac{K_{i^{\prime}}}{1-\gamma_{i^{\prime}} s} \equiv \prod_{i^{\prime}=1}^{u} \frac{1}{1-\gamma_{i^{\prime}} s}
$$

To obtain $K_{1}$, (72) is multiplied by $\left(1-\gamma_{1} s\right)$ to yield

$$
\left(1-\gamma_{1} s\right) \quad \sum_{i^{\prime}=1}^{u} \frac{K_{i^{\prime}}}{1-\gamma_{i^{\prime}} s} \equiv\left(1-\gamma_{1} s\right) \quad \prod_{i^{\prime}=1}^{u} \frac{1}{1-\gamma_{i^{\prime}} s}
$$

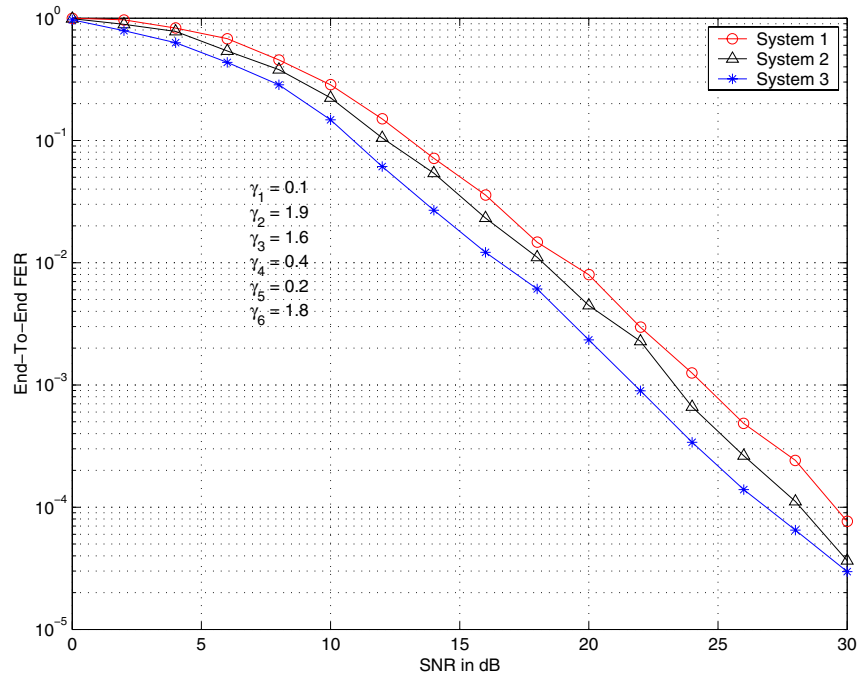

Fig. 7. Comparison between end-to-end frame-error rate of the three configurations for unequal sub-channel gains.

after which $\mathrm{s}$ is set to $s=1 / \gamma_{1}$ to arrive at

$$
K_{1}=\prod_{i^{\prime}=2}^{u} \frac{1}{1-\frac{\gamma_{i^{\prime}}}{\gamma_{1}}}=\prod_{i^{\prime}=2}^{u} \frac{\gamma_{1}}{\gamma_{1}-\gamma_{i^{\prime}}}
$$

The same procedure is repeated for any $K_{i}$ to derive (71).

\section{REFERENCES}

[1] M. Dohler, "Virtual antenna arrays," Ph.D. dissertation, University of London, United Kingdom, 2004.

[2] A. Sendonaris, E. Erkip, and B. Aazhang, "User cooperation diversity, part i:system description," IEEE Trans. Comm., vol. 51, no. 11, pp. 1927-1938, Nov. 2003.

[3] X. Li and N. E. Wu, "'Power efficient wireless sensor networks with distributed transmission-induced space spreading"," in Proc. 37th Asil. Conf. Signals, Syst., Comput., Pacific Grove, CA, Nov 2003.

[4] J. N. Laneman and G. W. Wornell, "Distributed space-time-coded protocols for exploiting cooperative diversity in wireless networks," IEEE Trans. Inform. Theory, vol. 49, no. 10, pp. 2415-2425, Oct. 2003.

[5] X. Li, "Space-time coded muli-transmission among distributed transmitters without perfect synchronization," IEEE Signal Processing Letters, vol. 11 , no. 12 , pp. 948-951, Dec. 2004.

[6] K. Tourki and L. Deneire, "Channel and delay estimation algorithm for asynchronous cooperative diversity," Springer's International Journal Wireless Personal Communications : Special Issue on Elective Topics towards Vision to Reality of the Wireless Future, vol. 37, no. 3-4, pp. 361-369, May 2006, invited paper.

[7] _ " "'Channel And Delay Estimation Algorithm For Asynchronous Cooperative Diversity"," in The 9th International Symposium on Wireless Personal Multimedia Communications, San Diego, CA, USA, Sep. 2006.

[8] _ , "'End-To-End Performance Analysis of Two-Hop Asynchronous Cooperative Diversity"," in 49th annual IEEE Global Telecommunications Conference, San Francisco, California, USA, Nov. 2006.

[9] S. M. Alamouti, "A simple transmit diversity technique for wireless communications," IEEE Journal on Select Areas in Communcations, vol. 16, no. 8, pp. 1451-1458, Oct. 1998.

[10] K. S. Marvin and M. S. Alouini., Digital Communication over Fading Channels, A Unified Approach to Performance Analysis. Wiley Series in Telecommunications and Signal Processing, 2000.

[11] H. Shin and J. H. Lee, “'"Exact Symbol Error Probability of Orthogonal Space-Time Block Codes"," in Proc. of the IEEE Global Telecommunications Conference., Taipei, Taiwan, Nov. 2002. 